\title{
Analytical solutions of the segregation problem
}

\author{
Andrey A. Pil'nik ${ }^{1, *}$, and Andrey A. Chernov ${ }^{1}$ \\ ${ }^{1}$ Kutateladze Institute of Thermophysics, 630090 Novosibirsk, Russia
}

\begin{abstract}
New analytical solutions of the segregation problem were found. The segregation is caused by the dissolved gas displacement by a moving crystallization front. The displacement is caused by the low solubility of the volatiles in solid, so the dissolved gas is withdrawn from the crystallizing melt towards the surrounding melt. Two different modes of crystallization were considered. The first one is the case of constant crystal growth rate which takes place in the case of intensive heat removal or when only initial stage of crystallization process is considered. The second mode is the "normal growth law" which is a self-similar solution of the crystal growth problem. In this case the crystal growth rate is inversely proportional to a square root of time. In contrast to the already existing solutions here the solidification shrinkage is taken into account.
\end{abstract}

\section{Introduction}

The crystallization processes are very common in the sphere of modern materials production. Often, solidified material contains pores and other imperfections. One mechanism of pore formation is the liquid rupture due to the solidification shrinkage [1]. Another mechanism of pore formation is the segregation during solidification. The melts often contain significant amount of dissolved volatiles and other impurities. Combined with the cooling regime these imperfections determine the final morphology of the sample $[2,3]$. Although, different methods of degassing and purification are available for the most cases it is not realistic to use them from the economical point of view. Most technological processes do not require the use of highly pure or homogeneous materials and depending on the tolerances even a pore formation to a limited extent can be acceptable. Of course some technological processes require the material completely without pores or at least impose strict constrains over the number of pores and other types of imperfections.

For the both cases it is highly important to be able to predict the conditions for pore formation caused by the segregation process during solidification. The pore formation depends on the dissolved gas concentration field during the whole process. So, the analytical solutions obtained in the article can be of great help for initial estimation of the cooling rate needed to achieve the desired level of porosity.

\footnotetext{
* Corresponding author: pilnik@,itp.nsc.ru
} 


\section{Problem statement}

We will consider the problem of segregation of dissolved gas by the moving plane crystallization front. The problem can be stated as the following diffusion problem.

$$
\frac{\partial C}{\partial t}+v_{l} \frac{\partial C}{\partial x}=D \frac{\partial^{2} C}{\partial x^{2}}
$$

where $t$ is the time; $x$ is the coordinate with the origin at the substrate surface; $C$ is the mass concentration of the volatile dissolved in melt; $D$ is the diffusion coefficient of the volatile dissolved in melt.

The equation (1) differs from the basic form of diffusion equation by containing the additional term caused by solidification shrinkage. Melt velocity $v_{l}$ can be found from the continuity equation. For the case of incompressible liquid $v_{l}=-(\bar{\rho}-1)(d X / d t)$, where $\bar{\rho}=\rho_{s} / \rho_{l}$ is the density ratio; $d X / d t$ is the crystal growth rate; $X$ is the crystallization front coordinate $(X=0$ at $t=0)$.

In the general case crystallization goes according to the following scenario [4,5]. During the first stage of the process (nonequilibrium crystallization) the supercooling of the melt near crystallization front is equal to the initial one, so the crystal growth is determined only by the kinetics of phase transition. Because of the latent heat of phase transition release the supercooling near the phase transition surface starts to decrease and asymptotically goes to zero. This stage can be labeled "equilibrium crystallization". Thermodynamic equilibrium establishes at the phase boundary (so called Stefan problem). In this case crystal growth is determined only by the heat transfer at the phase boundary and the crystal growth rate becomes inversely proportional to the square root of time.

At the inception of the process the dissolved gas concentration equals to $C_{0}$. We will study the case when $C_{s}<C_{0}<C_{l}$, where $C_{s}$ and $C_{l}$ are solubility in solid and solubility in liquid respectively.

The boundary conditions are derived from the conservation of the concentration at large distance from the crystallization front and the mass balance at the phase boundary:

$$
\begin{gathered}
(C)_{x \rightarrow \infty}=C_{0} ; \\
-D\left(\frac{\partial C}{\partial x}\right)_{x=X}=\bar{\rho} \frac{d X}{d t}\left[(C)_{x=X}-C_{s}\right] .
\end{gathered}
$$

Because of the fact that the range of coordinate $x$ changes in time it is problematic to use it in finding analytical solutions. Therefore, we will use coordinate characterized by the immobility of crystallization front: $\hat{x}=x-X$.

It is common while solving the heat and mass transfer problems to seek for the solution in dimensionless form, so we will be using following dimensionless variables: $\bar{C}=\frac{C-C_{0}}{C_{0}-C_{s}} ; \tau=t / t_{0} ; \bar{x}=x / x_{0} ; \bar{X}=X / x_{0} ; d \bar{X} / d \tau=v_{0}^{-1} d X / d t ; \delta=\bar{x}-\bar{X}$, where $t_{0}=D / v_{0}^{2}$ and $x_{0}=D / v_{0}$.

In the general form the formulated boundary value problem can only be solved numerically. But in certain cases of known crystal growth laws analytical solutions of segregation problem can be found. 


\section{Analytical solutions of the problem}

\subsection{The case of crystal growth rate inversely proportional to the square root of time}

Let us consider the case of "normal growth law". When the supercooling at crystallization front asymptotically goes to zero (equilibrium crystallization) and the crystal growth rate becomes inversely proportional to the square root of time [5]: $d X / d t=\beta_{T} \sqrt{a / t}$; $X(t)=2 \beta_{T} \sqrt{a t}$, where $a$ is the thermal diffusivity of the melt; $\beta_{T}=\beta_{T}(K u)$ is the dimensionless coefficient (a function of Kutateladze number $K u=L /\left(c \Delta T_{0}\right)$ ); $L$ is the specific heat of fusion; $c$ is the heat capacity; $\Delta T_{0}$ is the initial melt supercooling. In dimensionless form: $d \bar{X} / d \tau=\beta_{0} / \sqrt{\tau} ; \bar{X}=2 \beta_{0} \sqrt{\tau}$, where $\beta_{0}=\beta_{T} \sqrt{L e} ;$ Le $=D / a$ is the Lewis number.

Using dimensionless variables $\tau, \delta$ it becomes obvious that the solution of the problem becomes self-similar. Such solution but for the case of zero solidification shrinkage was obtained in [2]. Using variable $\eta=\delta / \sqrt{\tau}$ the diffusion equation can be reduced to the ordinary differential equation:

$$
\frac{d^{2} \bar{C}}{d \eta^{2}}+\left(\eta / 2+\beta_{0}^{*}\right) \frac{d \bar{C}}{d \eta}=0
$$

with boundary conditions

$$
\left(\frac{d \bar{C}}{d \eta}\right)_{\eta=0}=-\beta_{0}^{*}(\bar{C}+1) ;(\bar{C})_{\eta \rightarrow \infty}=0
$$

where $\beta_{0}^{*}=\bar{\rho} \beta_{0}$ is the only parameter depending on shrinkage appearing in equations describing the process. Solving this equation we can obtain:

$$
\bar{C}(\eta)=\frac{\sqrt{\pi} \beta_{0}^{*} \operatorname{erfc}\left(\eta / 2+\beta_{0}^{*}\right)}{\exp \left(-\beta_{0}^{* 2}\right)-\sqrt{\pi} \beta_{0}^{*} \operatorname{erfc}\left(\beta_{0}^{*}\right)}
$$

\subsection{The case of constant crystal growth rate}

Let us consider another case when the melt supercooling at the crystallization front stays the same during the crystallization process. Crystal growth rate depends only on the supercooling, so it also remains constant: $d X / d t=v_{0}=$ const ; $X=v_{0} t$ (in dimensionless form: $d \bar{X} / d \tau=1 ; \bar{X}=\tau$ ).

If solidification shrinkage is not taken into account the most obvious variables for the problem are $\tau$ and $\delta$ (so that the phase transition surface is immobile) as was done in [2]. Using these variables the problem can be stated in the following form:

$$
\frac{\partial \bar{C}}{\partial \tau}-\bar{\rho} \frac{d \bar{X}}{d \tau} \frac{\partial \bar{C}}{\partial \delta}=\frac{\partial^{2} \bar{C}}{\partial \delta^{2}}
$$




$$
\left(\bar{C}^{*}\right)_{\tau=0}=0 ;\left(\frac{\partial \bar{C}}{\partial \delta}\right)_{\delta=0}=-\bar{\rho} \frac{d \bar{X}}{d \tau}\left[(\bar{C})_{\delta=0}+1\right] ;(\bar{C})_{\delta \rightarrow \infty}=0
$$

The use of the variables $\tilde{\tau}=\bar{\rho}^{2} \tau, \tilde{\delta}=\bar{\rho} \delta$ allows us to bring the equations to the form they had in the case of zero shrinkage. So, this substitution allows to take the shrinkage into account without any additional complications.

By making a substitution $\bar{C}^{*}=\bar{C} \exp (\tilde{\tau} / 4+\tilde{\delta} / 2)$ the convective term can be excluded from the diffusion equation (7). The boundary value problem transforms into the following form:

$$
\begin{gathered}
\frac{\partial \bar{C}^{*}}{\partial \tilde{\tau}}=\frac{\partial^{2} \bar{C}^{*}}{\partial \tilde{\delta}^{2}} \\
\left(\bar{C}^{*}\right)_{\tilde{\tau}=0}=0 ;\left(\frac{\partial \bar{C}^{*}}{\partial \tilde{\delta}}\right)_{\tilde{\delta}=0}=-\bar{C}^{*} / 2-\exp (\bar{\tau} / 4) ;\left(\bar{C}^{*}\right)_{\tilde{\delta} \rightarrow \infty}=0 .
\end{gathered}
$$

The exact solution of the problem can be found using the Laplace transformation. The solution has the following form:

$$
\begin{aligned}
& \bar{C}(\tau, \delta)=\bar{\rho} \sqrt{\frac{\tau}{\pi}} \exp \left(-\frac{\delta^{2}}{4 \tau}-\frac{\bar{\rho} \delta}{2}-\frac{\bar{\rho}^{2} \tau}{4}\right)+ \\
& +\frac{1}{2}\left(1-\bar{\rho} \delta+\bar{\rho}^{2} \tau\right) \exp (-\bar{\rho} \delta) \operatorname{erfc}\left(\frac{\delta}{2 \sqrt{\tau}}-\frac{\bar{\rho} \sqrt{\tau}}{2}\right)-\frac{1}{2} \operatorname{erfc}\left(\frac{\delta}{2 \sqrt{\tau}}+\frac{\bar{\rho} \sqrt{\tau}}{2}\right) .
\end{aligned}
$$

\section{Conclusions}

The problem of dissolved gas segregation during solidification was formulated. The case of the plane crystallization front was studied. Solidification shrinkage was taken into account. Exact solutions of the problem were found for two different cases of crystal growth law: the constant crystal growth rate (non-equilibrium crystallization) and the case when crystal growth rate is inversely proportional to the square root of time (equilibrium crystallization). The obtained solutions allow us to understand the dissolved gas concentration field dynamics. In the case of equilibrium crystallization the solution is a self-similar one. It was shown that the obtained solutions are on the same complexity level as the solutions for the case of zero solidification shrinkage. Therefore, there is no justification for omitting the shrinkage from the equations as it was done traditionally.

The study was funded by FASO Russia.

\section{References}

1. A. A. Chernov, A. A. Pil'nik, Int. J. Heat Mass Transfer 55 (2012)

2. A. I. Fedorchenko, A. A. Chernov, Int. J. Heat Mass Transfer 46 (2003)

3. A .A. Chernov, A. A. Pil'nik, D. R. Islamov, J. Cryst. Growth 450 (2016)

4. A. A. Chernov, J. Eng. Thermophys. 18 (2009)

5. A. A. Chernov, A. A. Pil'nik, JETP Lett. 102 (2015) 